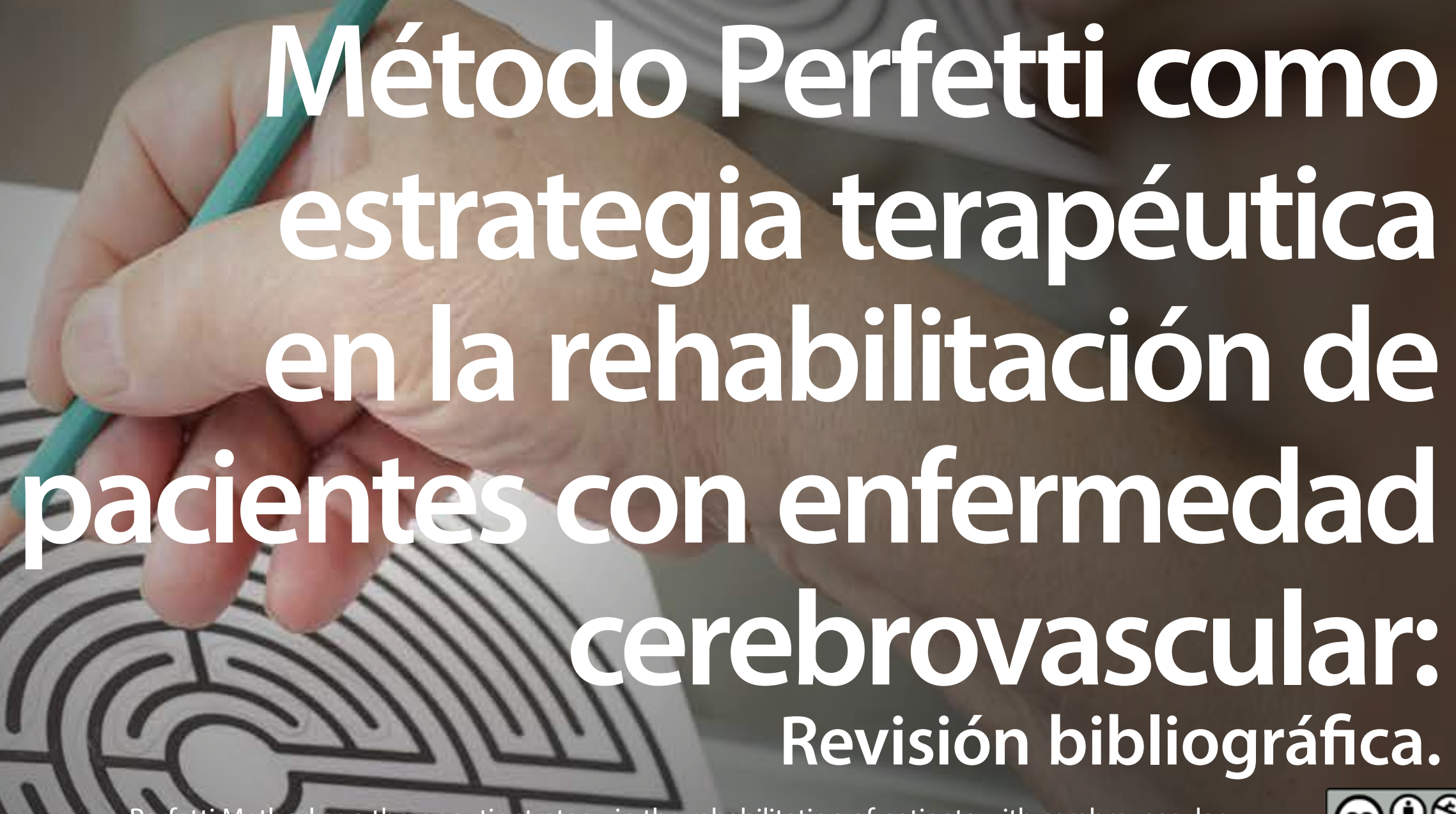

Perfettimethod as a therapeutic strategy in the rehabilitation of patients with cerebrovascular
disease: Bibliographic review.

(c) $) \Theta \Theta$

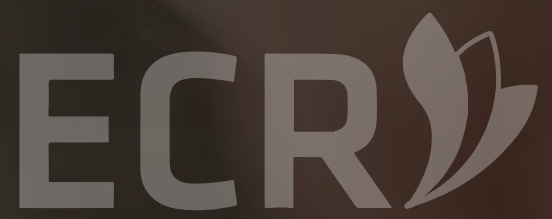




\section{Movimiento Cientifico}

ISSN-I: 2011-7197 | e-ISSN: 2463-2236 Publicación Semestral

2011-7191.mct.13107

itle: $\quad$ Perfetti method as a therapeutic strategy in the rehabilitation of patients with cerebrovascular disease: Bibliographic review.

ítulo: Método Perfetti como estrategia terapéutica en la rehabilitación de pacientes con enfermedad cerebrovascular: Revisión bibliográfica.

It Title / Título alternativo:

n]: $\quad$ Perfetti Method as a therapeutic strategy in the rehabilitation of patients with cerebrovascular disease: Bibliographic review.

s]: Método Perfetti como estrategia terapéutica en la rehabilitación de pacientes con enfermedad cerebrovascular: Revisión bibliográfica.

uthor (s) / Autor (es):

íaz Castro y Rodríguez López

eywords / Palabras Clave:

n]: Perfetti Method, Motor Function,

Cerebrovascular Disease,

Rehabilitation, Strategies.

s]: Método Perfetti, Función Motora,

Enfermedad Cerebrovascular,

Rehabilitación, Estrategias.

ubmited: $\quad$ 2018-12-10

cepted

2019-03-20

\section{Resumen}

Introducción: El método Perfetti, es un método de rehabilitación neurocognoscitivo el cual funciona sobre el sistema sensoriomotor. El objetivo del presente artículo fue hacer una revisión bibliográfica de las estrategias de intervención de este método y su aplicación en personas con alteraciones en miembro superior secundarias a enfermedad cerebrovascular.(ECV)

Método: Se realizó una búsqueda bibliográfica de 50 artículos, de los cuales 18 cumplieron con los criterios de inclusión entre los cuales se encontraban: el rango de año de publicación, método y patología Resultados: Teniendo en cuenta el análisis de los diversos artículos, se describen de los 18 seleccionados dos grupos: 8 artículos que contienen datos históricos del método, anatómicos y biomecánicos nutriendo así la introducción del presente artículo y el segundo grupo describe casos clínicos los cuales sustentan el objetivo de trabajo, la aplicación del método, las variables utilizadas en el método y las técnicas de terapia física. Conclusiones: La evidencia de los estudios y la recopilación de información respecto al método Perfetti es amplia en las bases teóricas, pero son pocos los estudios

enfocados en las alteraciones de miembro superior dadas por enfermedad cerebrovascular, debido a las condiciones en las cuales se debe realizar la aplicación del método, lo cual dificulta la obtención de datos más específicos que faciliten o promuevan futuras investigaciones en este campo.

\section{Citar como:}

Díaz Castro, W. M. y Rodríguez López, Y. C. (2019). Método Perfetti como estrategia terapéutica en la rehabilitación de pacientes con enfermedad cerebrovascular: Revisión bibliográfica. Movimiento Científico, 65-70.

\section{Abstract}

Introduction: The Perfetti Method is a method of neurocognitive rehabilitation which works on the sensorimotor system. The aim of this article was to make a systematic review of the intervention strategies of this method and its application in people with upper limb alterations secondary to cerebrovascula disease. (CVD) Method: A literature search of 50 articles was carried out, of which 18 met the inclusion criteria among which were: the range of year of publication, method and pathology Results: Taking into account the analysis of the various articles, two of the 18 selected are described: 8 articles that contain historical data of the method, anatomical and biomechanical, thus nourishing the introduction of this article and the second group describes clinical cases which suppor the objective of the work, the application

of the method, the variables used in the method and the physical therapy techniques.

Conclusions: The evidence from the studies

and the collection of information regarding the Perfetti method is broad in the theoretical basis, but few studies focused on the upper

limb alterations due to cerebrovascular disease, due to the conditions in which it must be performed. The application of the method, which makes it difficult to obtain more specific data that facilitate or promote future research in this field
Wendy Marcela Díaz Castro, Ft.

Source | Filiacion:

Escuela Colombiana de Rehabilitación

BIO:

Fisioterapeuta.

Estudiante de Especialización en Neurorehabilitación.

Escuela Colombiana de Rehabilitación.

City | Ciudad:

Bogotá [co]

e-mail:

marcdiaz2608@gmail.com 


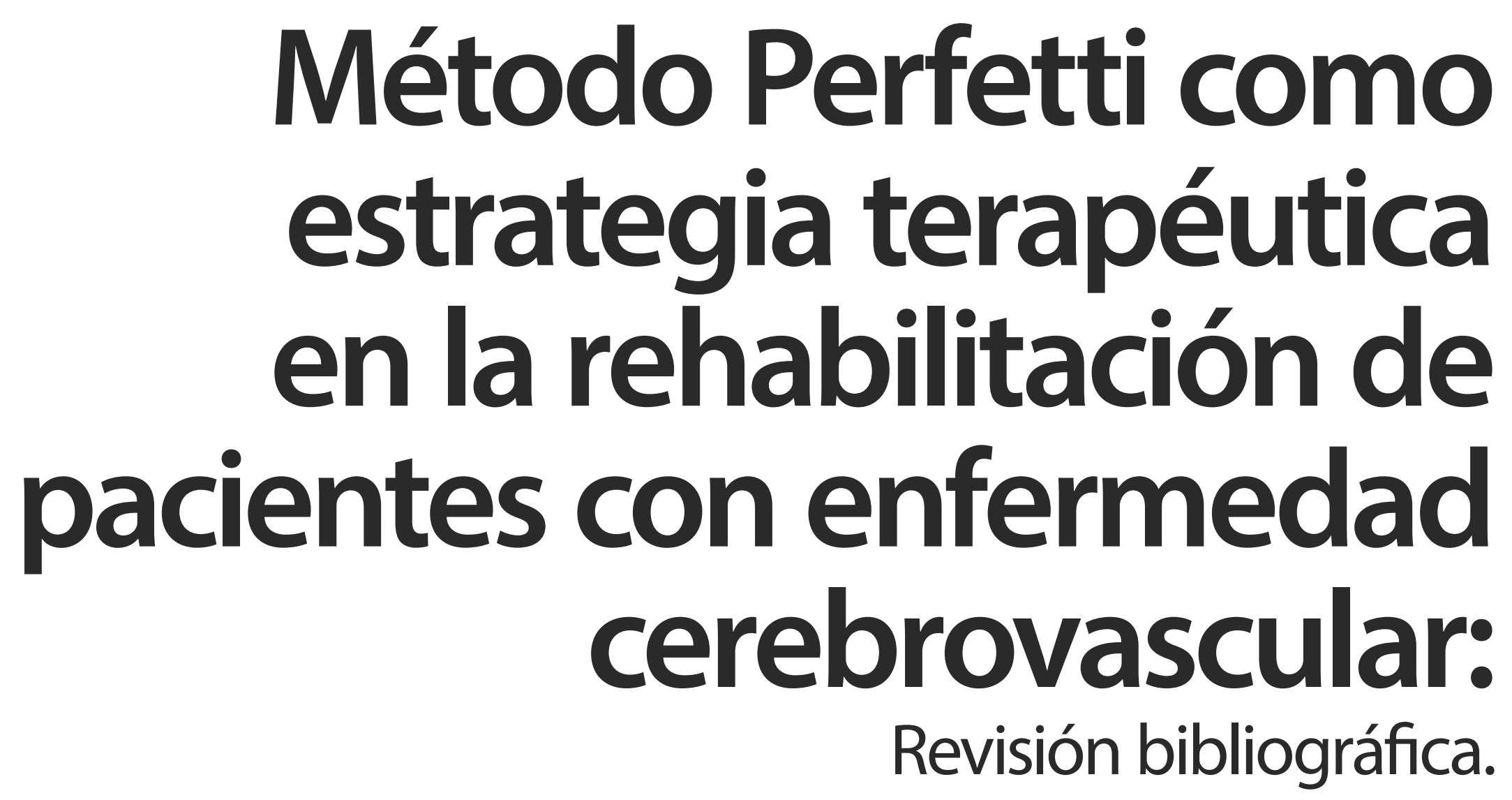

Perfetti Method as a therapeutic strategy in the rehabilitation of patients with cerebrovascular disease: Bibliographic review.

Wendy Marcela Díaz Castro Yuly Carolina Rodríguez López

\section{Introducción}

El método Perfetti o también llamado en la actualidad ejercicio terapéutico cognoscitivo, fuecreado porel neurólogo italiano Carlo Perfetti en los años 70, siendo una estrategia que presenta como base contextual, los diferentes principios neurocognitivos y tiene como objetivo la recuperación del movimiento de los pacientes con algún déficit neurológico, trabajando la reagrupación de estrategias basadas en experiencias, repeticiones, reorganización, atención, sentidos, percepción y reestructuración, con la finalidad de realizar un proceso de aprendizaje motor enfatizado en las necesidades y requerimientos del usuario (Centro Perfetti Neurorehabilitacion, s.f.). Este método inicialmente se enfatizó en los procesos de rehabilitación de la mano, Perfetti realizó un estudio tomando como punto de referencia el área cortical del ser humano donde identificó que la mano presenta alta complejidad tanto estructural como biomecánicamente, señalando como el órgano táctil por excelencia debido a los diversos receptores cutáneos que se encuentran en ella, además de ser una estructura vital para el desarrollo de la motricidad fina (Bonito Gadella, Martinez Fuentes y Martinez García, 2005).

A través del tiempo esta estrategia terapéutica evolucionó con la finalidad de ser un método funcional y global, trabajando el control secuencial progresivo, en donde se enfatiza en la rehabilitación de manera gradual tanto en la exploración y la planificación del movimiento como en su ejecución, facilitando la función motora del miembro superior e incluyendo de manera fundamental los patrones funcionales los cuales en conjunto con el área cognitiva permitirán el desarrollo adecuado de una tarea específica o ya programada (Azevedo Cacho Enio Walker, 2004) 
Según lo mencionado anteriormente, se evidencia que el compromiso funcional de miembro superior más significativo se da con mayor incidencia en la enfermedad cerebrovascular (Bernal, 2009), casi el 50\% de las admisiones neurológicas en los hospitales generales se deben a alguna forma de ECV; Se entiende como enfermedad cerebrovascular a un grupo heterogéneo de condiciones patológicas cuya característica común es la disfunción focal del tejido cerebral por un desequilibrio entre el aporte y los requerimientos de oxígeno y otros substratos. Incluye también las condiciones en las cuales el proceso primario es de naturaleza hemorrágica (Muñoz Collazos, 2011). Entre las secuelas más comunes se encuentra la hemiplejia, la cual se puede definir como la alteración y/o desequilibrio reflejo en donde no se encuentra el tono muscular lo suficientemente alto para vencer la gravedad ni los suficientemente bajo para poder facilitar un movimiento, cabe aclarar que el tipo de estabilidad muscular varía según las condiciones y los factores externos en los que se vean involucrados las personas que presentan esta condición de salud (Arce Enrique, 2010). Basados en diferentes referentes bibliográficos se precisa interpretar los diferentes medios y ejercicios que se aplican del método Perfetti en el proceso de rehabilitación en las personas que presentan enfermedad cerebrovascular, enfatizando en el déficit funcional de miembro superior, enmarcando en la importancia de promover la rehabilitación por medio de la función motora y el aprendizaje con actividades que asimilen vivencias y actividades de la cotidianidad (McEwen Sara, 2009)

\section{Materiales y métodos}

Se realiza una revisión bibliográfica recopilando diferentes fuentes bibliográficas, teniendo como criterios de inclusión: artículos publicados desde el año 2000 hasta el presente, que contengan los siguientes términos o palabras clave: método Perfetti, función motora, enfermedad cerebrovascular, rehabilitación, biomecánica y tren superior, se realiza también una búsqueda en inglés con términos como: Rehabilitation, Method Perfetti and Stroke, se utilizan como motores de búsqueda: PubMed, Scielo, Dialnet, ProQuest, EBSCO, Cochrane y Science Direct, de los cuales se recopilan 50 artículos viables, los mismos son integrados a una matriz de datos en la cual se incluyen los siguientes datos de cada artículo: título de artículo, nombre del autor, fecha de publicación, país, idioma, revista, factor de impacto, base de datos, nivel de evidencia, resumen, objetivos, metodología, resultados, conclusiones y el respectivo link. De la matriz anteriormente descrita, se realiza un filtro de los documentos encontrados, seleccionando 18 , de los cuales se tuvo en cuenta que presentaran características precisas como: la aplicación de una escala para evaluar, el tipo de estudio, preferiblemente que fuera una revisión sistemática e identificación de los hallazgos encontrados en cada estudio después de la aplicación del método Perfetti en esta población. Se realiza el análisis de los resultados de cada estudio y una comparación de los objetivos, enfoques y ejercicios, con la finalidad de describir la eficacia e importancia de implementar este tipo de métodos de manera constante en las intervenciones de esta población para garantizar una rehabilitación funcional e integral del paciente.

\section{Resultados}

Se realiza un filtro de información de los 50 artículos seleccionados de los cuales solo cumplen con los criterios 18 artículos en los mismos se identifica: que presentan fechas de publicación desde el año 2003 hasta el año 2017, con países de origen: Cuba, España, Estados Unidos, Reino Unido, México, Colombia, Alemania, El Salvador, Japón y Suiza, los cuales fueron hallados en bases de datos tales como: Pubmed, Scielo, Dialnet y Elsevier.

En primer lugar, se evidencia que según la ubicación geográfica el método Perfetti es conocido de diferentes maneras, tales como: tratamiento intensivo, técnica neurorehabilitadoras, mecanismos de neuroplasticidad y aplicación de un único método presentando todos unos mismos objetivos de estudio y se fundamentan en los principios básicos de la Neurorehabilitación (Masahiro Abo, 2012)

De los 18 artículos seleccionados, 8 de estos se basan en revisiones sistemáticas abarcando antecedentes históricos del método en mención, la biomecánica y la funcionalidad de miembro superior y su eficacia en la intervención, todos los artículos coinciden en que una de las alteraciones funcionales de mayor relevancia e incidencia es la hemiplejia definida como la alteración en el tono muscular, promoviendo un acortamiento de las fibras musculares por flexión involuntaria del hemicuerpo afectado, provocando cambios en la biomecánica lo que compromete la funcionalidad del miembro superior esto a su vez juega un papel importante en el rendimiento de tareas específicas o actividades de la vida diaria e instrumental ya que se ven estrechamente relacionadas con la participación en actividades sociales (Ochoa Padias, 2014)

Los 10 artículos restantes, se basan en estudios de caso con población que presentó las siguientes características: enfermedad cerebrovascular, edades entre 45 y mayores de 60 años y con compromiso funcional de miembro superior.

Al comparar las investigaciones, se evidencia que todos los estudios realizan en un primer momento el desarrollo de una valoración física, en donde tienen en común la recopilación de datos personales del usuario e indicadores primordiales del estado físico valorando: tono muscular, desempeño muscular, funcionalidad, rangos de movimiento y sensibilidad, coincidiendo de igual manera con las pruebas y escalas tales como: Fugl-Meyer, prueba de función manual (MFT),registro de actividad motora (MAL) (Sanchez Israel, 2009), por otra parte se identificó que en el momento de evaluar en algunos estudios se presentan mayor cantidad de test aplicados, los cuales son utilizados para confirmar los datos recolectados inicialmente.

Al momento de analizar los resultados se observan las siguientes similitudes: todas las intervenciones describen que las sesiones presentaban un tiempo aproximado de 30 a 40 minutos, donde se trabaja con grupo experimental y de control, proponiendo el método Perfetti como estrategia de primera elección para todas las investigaciones, en donde se implementa el desarrollo de ejercicios cognitivos por medio de subsidios, actividades funcionales, tareas de la vida diaria, realización de actividades kinésicas reconociendo el complejo articular del miembro superior y estimulación sensorial y propioceptiva (Tórtola Martín, 2015) 
A la descripción de los grupos control en los cuales se identifica la flexibilidad y variabilidad de estrategias de intervención desde el área de terapia física, se reconocen procesos como el trabajo postural, la modulación el tono muscular, ejercicios activos y asistidos para aumento de grados de libertad, la optimización del desempeño muscular, disminución de las retracciones musculares y técnicas de terapia manual (Rodriguez Lazaro Alvaro, 2016)

\section{Discusión}

En primer lugar se interpreta que la búsqueda de información, las investigaciones realizadas y la aplicación de dicho método en una patología como lo es la enfermedad cerebrovascular presenta diversas falencias respecto al tratamiento ya que la mayoría de autores basan su rehabilitación con diferentes técnicas desde las fases agudas del accidente cerebrovascular (Ángel, 2009) esto con la finalidad de obtener una rehabilitación eficaz y con una funcionalidad más amplia en el usuario, por ende como el criterio de inclusión principal de la investigación fue la fase crónica se redujeron ampliamente los estudios puntuales lo que ocasionó que el primer filtro de la información quedará corto en datos.

En segunda instancia el grupo de artículos encontrados, previamente filtrados en una base de datos y continuamente seleccionados presentó una amplia gama geográfica de lugares en los cuales se realizan las respectivas investigaciones, dejando en claro que el ECV es una patología con altos índices de padecimiento en la población de manera mundial (Moyano V, 2010), se denota que en un bajo número de artículos de la matriz se habla de estadísticas o porcentajes de la población que sufren los signos clínicos de la patología, lo que debería mayormente ser estudiado siendo de gran importancia a la hora de querer realizar la interpretación epidemiológica de la eficacia de la terapia física o de un método en específico como lo es Perfetti (Chanubol R, 2012)

Como punto importante y concordando con la información anteriormente descrita se identifica que de igual manera como esta patología se presenta en cualquier parte del mundo, la variabilidad de terminología precisando en el método Perfetti, puede crear confusión en caso de no conocer los principios y finalidades de este (Sunghee Lee, 2015), demostrando que es de gran importancia estandarizar el nombre del método.

Al puntualizar en los resultados obtenidos después de filtrar la información se identifican puntos importantes a discutir como lo son: la asertividad de los artículos a la hora de exponer signos clínicos, parámetros de evaluación, intervención y manejo de los usuarios quienes presentan ECV lo que promueve a la confiabilidad de la información y la poca variabilidad que se puede presentar (Devesa Gutierrez Ignacio, 2014)

A la hora de analizar los resultados que se obtuvieron a la aplicación de técnicas precisas y directas de la fisioterapia en ECV coinciden claramente en los procesos de rehabilitación, fluctuando en el paso a paso siendo totalmente comprensible ya que en cada lugar de aplicación de intervención presentan protocolos, normal o diferentes maneras de intervención (Samar M. Hatem, 2016)
Con respecto al análisis de la aplicación del método Perfetti en la población con ECV se identifica que al contrario de la terapia convencional física este método presenta una gama diferente de objetivos conllevando a diversidad en estrategias de trabajo, ya que su área predominante a fortalecer es la cognitiva lo que desencadena respuestas motoras y así evidenciando tareas conjuntas tanto mentales como físicas (Domínguez Ferraz, Da Silva-Ribeiro, De Matos-Pinheiro y Pedreira-da Fonseca, 2014)

De los artículos revisados se afirma que todos los métodos utilizados tanto los convencionales como los nuevos el también conocido como ejercicio terapéutico cognoscitivo o Método Perfetti presenta mejores resultados y más evolución en la recuperación motora de miembro superior, se manifiesta que entre más tiempo se hace más difícil recuperar las funciones distales de alta complejidad, entre las actividades motoras se destaca el agarre cilíndrico que es una tarea de baja complejidad aunque aún falta que se realicen más estudios que analicen estas variables se ha evidenciado mejoría significativa en los pacientes con enfermedad cerebrovascular.

\section{Conclusiones}

En la enfermedad cerebrovascular se evidencia en sus antecedentes clínicos las alteraciones físicas y cognitivas dadas como consecuencia de los cambios neurofisiológicos, entre ellas se encuentra la fluctuación del tono muscular con mayor relevancia en extremidades superiores, provocando la incapacidad para ejecutar actividades de manera independiente y habilidosa, por tanto es de gran importancia implementar procesos de rehabilitación de manera temprana, las cuales sean flexibles y desarrollen diversas estrategias de intervención de tipo integral como se puede demostrar en el trabajo del método Perfetti.

Teniendo en cuenta lo anteriormente descrito y el análisis de la presente revisión, se concluye que la terapia física en el entorno de la rehabilitación global para usuarios con secuelas de enfermedad cerebro vascular debe mostrar variabilidad en su ejecución, técnicasy procedimientos (Pollock Alex, Peter Langhorne y Valerie M. Pomeroy, 2008).

La terapia física siendo una de las más solicitadas a la hora de la rehabilitación de esta población, basa su intervención en técnicas cinética, las cuales presentan amplios estudios verídicos, sin embargo, se encuentra que el desarrollo y la eficacia de la terapia al emplear métodos de Neurorehabilitación como lo es Perfetti promueven a que el desarrollo de las mismas exhiba una retroalimentación duradera y abarquen áreas físico cognitivas de modo más efectivo (Sallés Laia, 2017)

Perfetti como método neurorehabilitador se encuentra descrito como uno de los métodos más completos y eficaces a la hora de trabajar alteraciones en extremidades, no obstante, a la evidencia su aplicación en terapia se muestra escasa ya que los estudios no son suficientes y compleja por los factores externos que se deben tener en cuenta, como lo son: materiales, tiempo disponible y capacidad de atención.

Es recomendable aumentar el número de estudios sobre la aplicación del método Perfetti en la intervención de la población que muestre disfunciones o alteraciones en miembro superior 


\section{Método Perfetti como estrategia terapéutica en la rehabilitación de pacientes con enfermedad cerebrovascular}

enfocándose especialmente en personas que presentan secuelas de ECV, ya que es escasa la profundización de su veracidad y eficacia, es de gran importancia para el área de Neurorehabilitación el conocer la correcta ejecución de actividades, procesos y ejercicios en el desarrollo de la misma para así poder tener soportes científicos que validen las investigaciones y poder realizar una confiable aplicación.

\section{Referencias}

Ángel, A. C. (2009). Rehabilitación del ACV:evaluación, pronóstico y tratamiento. Galicia Clinica :Revista Oficial de Sociedad Galega de Medicina Interna ( SOGAMI ). Obtenido de https://galiciaclinica.info/pdf/5/81.pdf

Arce Enrique, M. J. (2010). Atención rehabilitadora de la enfermedad cerebrovascular en el municipo Artemisa. Revista Cubana de Medicina General Integral. Obtenido de http://scielo.s/d.cu/pdf/mgi/v26n2/mgi07210. $p d f$

Azevedo Cacho Enio Walker, V. d. (2004). Evaluación de la recuperación motora de pacientes hemipléjicos a través del protocolo de rendimiento fisico de Fugl-Meyer. Revista Neurociencias, 12(2). Obtenido de http://wWw. revistaneurociencias.com.br/edicoes/2004/RN\%2012\%2002.pdf\#page=37

Bernal, M. Y. (2009). Alteraciones de la función motora de miembro superior en la hemiplejía -modelos de intervención fisioterapéutica. Revista Movimiento Cientifico.

Bonito Gadella, J. C., Martinez Fuentes, J. y Martinez García, R. (2005). El ejercicio terapéutico cognoscitivo: Concepto Perfetti. Revista de fisioterapia, 4(1), 36-42.

Centro Perfetti Neurorehabilitacion. (s.f.). Obtenido de El método Perfetti: https:// www.centroPerfetti.com/metodo-Perfetti

Chanubol R, W. P. (2012). A randomized controlled trial of Cognitive Sensory Motor Training Therapy on the recovery of arm function in acute stroke patients. Clinical Rehabilitation. Obtenido de https://www.ncbi.nlm.nih.gov/ pubmed/22649162

Devesa Gutierrez Ignacio, M. G. (2014). Rehabilitación del paciente con enfermedad vascular cerebral (EVC). Revista Mexicana de Medicina Fisica y Rehabilitacion. Obtenido de http://wwW.medigraphic.com/pdfs/fisica/mf2014/mf143 4e.pdf

Domínguez Ferraz, D., Da Silva-Ribeiro, N. M., De Matos-Pinheiro, I. y Pedreirada Fonseca, E. (2014). Eficacia del método Perfetti en el tratamiento de secuelas del accidente cerebrovascular: una revisión sistemática. Cuestiones de fisioterapia: revista universitaria de información e investigación en Fisioterapia, 43(3), 196-205. Obtenido de https://dialnet. unirioja.es/servlet/articulo?codigo $=5435547$

Masahiro Abo, W. K. (2012). Rehabilitation for Cerebrovascular Disease:Current and new methods in Japan. JMAJ Japan Medical Association Journal. Obtenido de https://www.ncbi.nlm.nih.gov/pubmed/25237224

McEwen Sara, H. M. (2009). Cognitive strategy use to enhance motor skill acquisition post-stroke: A critical review. Brain Injury. Obtenido de https://www.tandfonline.com/doi/ abs/10.1080/02699050902788493?journalCode=ibij20

Moyano V, A. (2010). El accidente cerebrovascular desde la mirada del rehabilitador. Revista Hospital Clinico Universidad de Chile. Obtenido de https://www.redclinica.cl/Portals/0/Users/014/14/14/Publicaciones/Revista/ accidente cerebrovascular desde mirada rehabilitador.pdf

Muñoz Collazos, M. (2011). Enfermedad Cerebrovascular. Guía Neurologica 1, 205-237. Asociación Colombiana de Neurología. Obtenido de https://www. acnweb.org/guia/glc12i.pdf

Ochoa Padias, D. S. (2014). Beneficios del método Perfetti aplicado en miembro superior hemipléjico en pacientes con diagnostico de evento cerebro vascular, que asisten al centro de rehabilitacion Integral de Oriente. Tesis de Grado para Licenciatura en Fisioterapia y Terapia Ocupacional. Obtenido de http://ri.ues.edu.sv/7389/

Pollock Alex, G. D., Peter Langhorne y Valerie M. Pomeroy, P. M. (2008). Physiotherapy Treatment Approaches for Stroke. Journal of the American Heart Association. Obtenido de https://www.ahajournals.org/doi/ abs/10.1161/strokeaha.107.492710

Rodriguez Lazaro Alvaro, O. C. (2016). Cambios en la recuperación de la función motora en pacientes con accidente cerebrovascular crónico. IATREIA Revista Medica Universidad de Antioquia, 29(2). Obtenido de http:// www.scielo.org.co/scielo.php?pid=S0121-07932016000200002\&script=sci abstract\&tlng=es

Sallés Laia, C. P. (2017). A neurocognitive approach for recovering upper extremity movement following subacute stroke:a randomized controlled pilot study. The Journal of Physical Therapy Science. Obtenido de https://www.ncbi. nlm.nih.gov/pmc/articles/PMC5430270/pdf/jpts-29-665.pdf

Samar M. Hatem, G. S. (2016). Rehabilitation of Motor Function after Stroke: A Multiple Systematic Review Focused on Techniques to Stimulate Upper Extremity Recovery. Frontiers in Human Neuroscience. Obtenido de https:// www.frontiersin.org/articles/10.3389/fnhum.2016.00442/full

Sanchez Israel, H. J. (2009). Actividades simuladas de la vida diaria para rehabilitación motora del miembro superior en pacientes con enfermedad vascular cerebral. Archivos de Neurociencias, 14(4). Obtenido de http:// www.medigraphic.com/pdfs/arcneu/ane-2009/ane094e.pdf

Sunghee Lee, S. B. (2015). The effects of cognitive exercise therapy on chronic stroke patients' upper limb functions, activities of daily living and quality of life. Journal of Physical Therapy Science. Obtenido de https://www.ncbi.nlm. nih.gov/pmc/articles/PMC4616095/pdf/jpts-27-2787.pdf

Tórtola Martín, N. (2015). Método Perfetti para el tratamiento de la hemiplejía desde terapia ocupacional. Revista de Terapia Ocupacional de Galicia, $1(10), 2$. 